\title{
LA REVISTA PERUANA DE MEDICINA EXPERIMENTAL Y SALUD PÚBLICA CONTINÚA AL SERVICIO DE LA SALUD PÚBLICA DEL PERÚ Y LATINOAMÉRICA
}

\author{
THE PERUVIAN JOURNAL OF EXPERIMENTAL MEDICINE AND PUBLIC HEALTH \\ CONTINUES TO SERVE THE PUBLIC HEALTH OF PERU AND LATIN AMERICA
}

\author{
Zuño Burstein ${ }^{1, a}$, Cesar Cabezas ${ }^{1, b}$
}

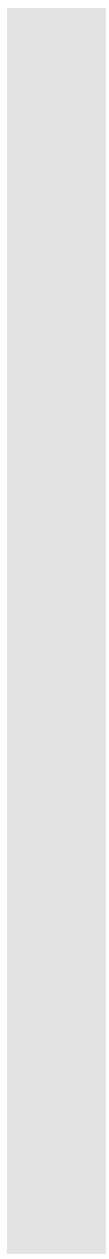

La Revista Peruana de Medicina Experimental y Salud Pública (RPMESP) órgano oficial de difusión científica del Instituto Nacional de Salud (INS), brazo científico del Ministerio de Salud (MINSA) del Perú, se encuentra frente a un nuevo año (2016) con importantes cambios institucionales, que podrían comprometer en forma impredecible toda la actividad y panorama médico en todos sus aspectos y manifestaciones; esperamos que estos no afecten las actividades y proyecciones de la Revista.

Estos cambios comprenden las elecciones para nuevas autoridades gubernamentales, comenzando con los poderes Ejecutivo y Legislativo. Es digno de mencionar que desde el inicio del actual gobierno, la RPMESP estableció el compromiso de colaborar con el éxito de su gestión gubernamental, tal como lo ha hecho con los gobiernos anteriores ${ }^{(1)}$, en el área de su competencia, en beneficio de la salud pública y el bienestar de nuestro país ${ }^{(2)}$. Igualmente, las instituciones representativas de los médicos peruanos, la Academia Nacional de Medicina (ANM) y el Colegio Médico del Perú (CMP), han cambiado sus cuerpos directivos con procesos electorales democráticos y, finalmente, nuestra gestión bianual en la dirección y otros cargos en el Comité de la Revista ha terminado a fines del 2015 y ha sido renovada recientemente por el Periodo 2016-2018.

En la comunicación dirigida por el director de la Revista al Dr. Aníbal Velásquez Valdivia, actual ministro de Salud (exdirector del INS y miembro del Consejo Consultivo de la RPMESP) adjuntando el "Informe de Gestión de la Revista Peruana de Medicina Experimental y Salud Pública - 2015", y que fuera presentado al actual jefe del INS, Dr. Ernesto Gozzer Infante, se manifiesta textualmente que: Nuestra Revista, como se señala en el informe adjunto, está calificada como la mejor revista médico-científica peruana y una de las mejores de Salud Pública y de origen estatal, a nivel latinoamericano; siendo el producto de un esfuerzo mancomunado de un grupo humano profesional, profundamente identificado con su trabajo de promover y difundir información seria, basada en investigaciones científicas calificadas, sobre problemas de salud pública, predominantemente latinoamericana, aplicada a nuestro país y que en acciones conjuntas con la Academia Nacional de Medicina, institución asesora de los poderes públicos en problemas de Salud, se hace llegar a los investigadores, académicos, ejecutores del campo médico y a los decisores políticos gubernamentales en beneficio de nuestro país".

La RPMESP es el producto de un indesmayable esfuerzo que ha sido comunicado periódicamente en sucesivos editoriales de esta publicación ${ }^{(3-8)}$ defendiendo la necesidad de la investigación y su difusión

\footnotetext{
Revista Peruana de Medicina Experimental y Salud Pública, Instituto Nacional de Salud. Lima, Perú.

Director; ${ }^{b}$ editor general

Recibido: 07-03-16 Aprobado: 09-03-16
}

Citar como: Burstein Z, Cabezas C. La revista peruana de medicina experimental y salud pública continúa al Servicio de la Salud pública del Perú y latinoamérica. [editorial]. Rev Peru Med Exp Salud Publica. 2016;33(1):10-2. doi: 10.17843/rpmesp.2016.331.2007 
en beneficio de la Salud Pública y del bienestar de la población en general ${ }^{(9)}$.

Es seria y preocupante la situación de crisis en el Perú y en el mundo, existe inestabilidad institucional, inseguridad, corrupción y quebrantamiento de valores fundamentales; en el área médica en nuestro país ello se expresa en la proliferación irracional de facultades de Medicina con marcadas limitaciones para formar médicos con los requisitos mínimos para desempeñar profesionalmente su servicio a la comunidad, con el principio básico de "medicina basada en la persona", así como escasa información actualizada y falta de capacitación médica permanente. Se aúna a ello otras deficiencias como: la mercantilización con excesivo interés de lucro por parte de los servicios de salud y de centros educacionales formadores de recursos humanos para la salud; la inexistencia de un control de precios de los medicamentos, que son excesivos, y un deficiente control de los medicamentos genéricos; la falta de una adecuada coordinación en las medidas preventivas de salud; el maltrato y la agresión a la dignidad médica por parte de autoridades administrativas gubernamentales y empresariales privadas, entre otros aspectos, males que tratan de ser subsanados con disposiciones legales como la "Reforma de Sector Salud" y la "Ley Universitaria", entre otras, pero que enfrentan, por intereses subalternos, dificultades en su aplicación para establecer la justa expectativa de la salud, la educación y la vivienda como derechos fundamentales de todo ciudadano.

Todo lo expresado, agregado a las amenazas de catástrofes naturales condicionadas por el cambio climático que se predice, afectará seriamente a nuestro país con repercusión en la salud pública en los próximos meses del presente año; por otra parte, la peligrosa aparición de epidemias de enfermedades transmitidas por mosquitos vectores, como el dengue, chikungunya, zika y otras afecciones emergentes y reemergentes, obligan a las instituciones como la Academia Nacional de Medicina y el Instituto Nacional de Salud(10), a intensificar su participación, cumpliendo la función que le corresponde al INS como ejecutor tecnológico, y de reserva moral y académica a la ANM, asesorando, además, a los responsables políticos sobre problemas de su competencia en beneficio de la comunidad.

En el afán de intervenir activamente en el nivel que le corresponde en los aspectos señalados, la RPMESP con su director y el Comité Editor de la Revista han establecido como política editorial, desde el año 2012, la designación para cada número de la Revista de un tema de importancia en la Salud Pública, con un editor invitado experto en el tema (11), para comprometer trabajos y artículos, que, sometidos al riguroso proceso editorial, han sido publicados y con la salida del número correspondiente se convoca a un simposio, conjuntamente con la ANM, para que con expertos invitados y público interesado se debata y se saquen conclusiones para hacerlas llegar a los poderes públicos.

Es así que se han tratado durante el año 2012 los temas "Violencia y Adicciones", "Salud Ocupacional", "Nutrición en el Perú" y "Ensayos Clínicos"; el año 2013: "Cáncer como problema de Salud Pública", "Atención Primaria de la Salud", "Salud Sexual y Reproductiva" e "Inequidad en Salud"; el año 2014: "Enfermedades Crónicas no Transmisibles", "Enfermedades Infecciosas desatendidas en el Perú y las Américas", "Formación de Recursos Humanos en Salud" y "Reforma del Sistema de Salud Peruano" y el año 2015: "Enfermedades Infecciosas Emergentes y Reemergentes" con el Dr. Daniel Bausch, "Informática Médica y Telesalud" con el Dr. Walter Curioso, "Desarrollo Infantil Temprano" con el Dr. Melitón Arce y "Ciencias Básicas y su Contribución a la Salud” con el Dr. Heinner Guio.

El grupo editor de la RPMESP ha realizado, como en años anteriores, cumpliendo la función de trasferencia tecnológica y asesoría para otras publicaciones médicocientíficas peruanas, y con el propósito de hacer docencia dirigida a editores médicos, un taller, el 29 de octubre de 2015, sobre revistas biomédicas peruanas y participado en un curso previo al Congreso Internacional del Instituto Nacional de Salud, el 10 de noviembre de 2015, sobre Investigación y Redacción Científica Biomédica.

La RPMESP ha cumplido estrictamente con su calendario de publicaciones, habiendo recibido durante el año 2015: 446 solicitudes de publicación para las diferentes secciones de la Revista de donde 354 fueron de autores nacionales y 92 de autores extranjeros de los siguientes países: México, Cuba, EE. UU., Colombia, Argentina, España, Venezuela, Ecuador, Brasil, Uruguay, Costa Rica, Chile y Paraguay; de ellos, fueron aprobados para su publicación 73 de autores nacionales y 15 de autores extranjeros.

La RPMESP queda abierta para la publicación de artículos que sean contribuciones a la salud pública, para sus diferentes secciones, además de los comprometidos y enviados para el tema principal de cada número, por los autores que cumplan con los requerimientos exigidos en las "Instrucciones para la Presentación de Artículos a la Revista Peruana de Medicina Experimental y Salud Pública", que se publican en cada número de la versión impresa de la Revista y en su página web, adjuntando los formatos correspondientes y que sean aprobados para su publicación después de pasar por el proceso editorial correspondiente, con la calificación por pares 
de expertos nacionales e internacionales, a quienes agradecemos por su valiosa colaboración. Es necesario tomar en cuenta que la RPMESP esta indizada en las bases de datos más exigentes y que desde el próximo número se publicará en idioma inglés, en versión electrónica y a texto completo.

Para el año 2016 se han programado y comprometido los siguientes temas: "Impacto del Cambio Climático sobre la Salud" con el Dr. Rubén Figueroa como editor invitado; para ello se ha solicitado artículos sobre el tema a expertos nacionales y extranjeros así como a las autoridades académicas, sanitarias y gubernamentales del Poder Ejecutivo y de las municipalidades; la recepción de artículos para este número se cierra el 22 de enero y será publicado el 21 de marzo. Para el segundo número: "Salud y Calidad de Vida en el Adulto Mayor" con el Dr. Luis Varela como editor invitado, el cierre de recepción de artículos es el 28 de febrero, para ser publicado el 16 de mayo. El siguiente es "Obesidad como Problema de Salud Pública" con el Dr. Miguel Malo como editor invitado, el cierre de recepción de artículos es el 25 de mayo, para ser publicado el 11 de julio y, por último, "Medicina Centrada en la Persona: Ciencia y Humanismo" con el Dr. Alberto Perales como editor invitado, el cierre de recepción de artículos es el 03 de junio, para ser publicado el 15 de septiembre. Las fechas previstas para la realización de las conferencias conjuntas con la ANM, serán los días 7 de abril, 7 de julio, 6 de octubre y 7 de diciembre, respectivamente para cada uno de los temas señalados y después de haber sido publicado en su versión impresa el número correspondiente de la Revista.

Es un reto para la RPMESP el emprender nuevas actividades en este año iniciando la publicación anticipada, videos de las presentaciones conjuntas con la ANM, videos en la galería fotográfica y un aspecto importante como es el compartir los conocimientos publicados en la RPMEPS con el ciudadano, en un lenguaje asequible a través de los medios de comunicación masiva; para este propósito y la difusión de la RPMESP y su quehacer contamos con el apoyo complementario del CONCYTEC.

Con la seguridad de haber cumplido con la importante misión encomendada y dejar para la nueva gestión el terreno allanado para la continuación de esta importante publicación estatal al servicio del país y de la salud pública latinoamericana y contando con el respaldo y apoyo comprometido de la jefatura actual del Instituto, del Ministerio de Salud Pública, del Gobierno Central e instituciones comprometidas con nuestra labor, en nombre del Comité Editor de la Revista agradecemos la colaboración brindada.

\section{REFERENCIAS BIBLIOGRÁFICAS}

1. Burstein Z. Revista Peruana de Medicina Experimental y Salud Pública del Instituto Nacional de Salud. En: Historia de la Salud Pública en el Perú. Academia Peruana de Salud vol. 25 Secc II: Otros avances en la investigación histórica. Pag. 8983-96 JC Treiding Peru. SA 2013.

2. Burstein Z, Cabezas C. Revista Peruana de Medicina Experimental y Salud Publica: 70 años cumpliendo sus Metas y Proyectándose al Futuro. Rev Peru Med Exp Salud Publica. 2012;9(1):6-8

3. Cabezas C, Mayta-Tristán P. Evolución, Prospectivas de la Revista Peruana de Medicina Experimental y Salud Publica 1945-2008. Rev Peru Med Exp Salud Pública. 2008;25(2):167-168.

4. Burstein Z. Revista Peruana de Medicina Experimental y Salud Publica en el año 2009. Rev Peru Med Exp Salud Publica. 2009;26(1):3-4.

5. Burstein Z, Cabezas C. Avances y Retos de la Revista Peruana de Medicina Experimental y Salud Pública al 2010. Rev Peru Med Exp Salud Pública. 2010;27(1):3-5.

6. Burstein Z, Mayta-Tristán P. La Revista Peruana de Medicina Experimental y Salud Publica ahora indizada en MEDLINE/INDEX MEDICUS. Rev Peru Med Exp Salud Publica. 2010;27(3):312-14.

7. Burstein Z. Presentación pública de la versión actual y reseña histórica de la Revista Peruana de Medicina Experimental y Salud Pública. Rev Peru Med Exp y Salud Publica. 2011;28(1):8-12.
8. Cabezas C. Necesidad de la Investigación en Salud para contribuir a la equidad, la salud y el desarrollo. Rev Peru Med Exp Salud Pública. 2010;27(3):310-11.

9. Cabezas C, Arroyo-Hernández $\mathrm{H}$, Solari L. Revista Peruana de Medicina Experimental y Salud Pública su importancia en la difusión de la ciencia desde el Perú. Rev Peru Med Exp Salud Pública. 2015;32(3):421-2.

10. Burstein Z. La Academia Nacional de Medicina y el Instituto Nacional de Salud: Instituciones Tutelares de la Salud Publica y de la investigación medico científica en el Perú. Rev Peru Med Exp Salud Publica. 2013;30(1):9-11.

11. Burstein Z. Simposio y Números Temáticos en la Revista Peruana de Medicina Experimental y Salud Pública. 2008;25(1):5-6. 